\title{
L'espace des mobilisations contre les violences des forces de l'ordre en France depuis les années 1990
}

\section{Anthony Pregnolato ${ }^{1}$}

Depuis l'instauration et les prolongations de l'état d'urgence en France dès novembre 2015, puis le mouvement contre la « Loi travail » au printemps 2016, la question des violences policières est fortement médiatisée et politisée. Mais les violences des forces de l'ordre et leurs dénonciations sont loin d'être un phénomène récent. Dès les années 1960, des mobilisations émergent contre ces violences, principalement à l'encontre des travailleurs immigrés des anciennes colonies françaises et de leurs enfants. Dans les années 1970, des mobilisations dénoncent les violences policières touchant les jeunes héritiers de l'immigration post-coloniale habitant les cités de banlieues, et dans les années 1980 une nouvelle génération de militant-e-s dénonçant les « crimes racistes et sécuritaires » émerge, notamment lors de la Marche pour l'égalité et contre le racisme de 1983. À partir des années 1990, ces mobilisations connaissent une plus forte médiatisation et politisation. On retrouve alors souvent un même schéma : après la mort d'un habitant de cité (souvent « jeune », homme, héritier de l'immigration post-coloniale), des rébellions éclatent, s'en prenant aux forces de l'ordre et en brûlant des bus, des voitures, des écoles ou des bibliothèques, dénonçant par-là tant des rapports conflictuels entre les forces de l'ordre et les « jeunes » faisant l'objet de contrôles policiers réguliers, que des injustices ressenties et vécues par les franges les plus précarisées des classes populaires vivant dans ces cités. Des familles de victimes de violences policières et des militant-e-s des quartiers populaires mettent aussi en place d'autres modes d'action (manifestations, sit-in, créations de comités de soutien, de collectifs ou d'associations, actions en justice) et tendent à engager une mobilisation sur le temps long. Si ce n'est pas toujours le cas, des travaux ont montré qu'il pouvait y avoir une continuité d'acteur-rice-s d'un mode d'action à l'autre ${ }^{2}$. Si principalement les mobilisations contre les violences des forces de l'ordre émergent ainsi dans les quartiers populaires, d'autres organisations, ne trouvant pas leur genèse directement dans ce schéma, incluent dans leur mobilisation la dénonciation des déviances violentes des forces de l'ordre, telles que des organisations des quartiers populaires, des organisations antiracistes, des organisations d'extrêmegauche, ou des organisations de défense des droits de l'homme. L'ensemble de ces dénonciations d'un usage de la violence physique par les forces de l'ordre forme ce que l'on peut désigner un espace de mobilisations contre les violences des forces de l'ordre, dans la mesure où elles

1 Doctorant en science politique (ISP-Paris Nanterre / Centre Marc Bloch - Saisir l'Europe)

2 Dossier " Dammarie-les-Lys : les militants de l’incertitude », Vacarme, n²1, automne 2002 ; Hajjat, A., " Rébellions urbaines et déviances policières. Les “jeunes“ des Minguettes face à la police (1981-1983) », Cultures \& Conflits, 2014 
constituent une « configuration de collectifs - et de leurs [participant-e-s] $]^{3} »$, pris-es dans des « liens d’interdépendance qui peuvent relever de la concurrence (...), de la coopération (...), ou encore de l'opposition plus ou moins virulente ${ }^{4} »$, qui prennent pour objet de lutte, unique ou non, les violences des forces de l'ordre. Se demander comment se construit une cause contre les violences des forces de l'ordre, passe par l'interrogation des modes d'actions et des perceptions de ces dernières par les militant-e-s et les collectifs. Sans en faire une présentation exhaustive, l'article propose d'interroger ces différentes manières de lutter pour une compréhension de cet espace de mobilisations depuis les années 1990. Ce dernier est traversé par cinq pôles militants qui se différencient les uns des autres par des modes d'actions privilégiés et des perceptions différentes de la cause. Si ces dernières donnent lieux à de fortes divisions, un-e militant-e ou un collectif peut naviguer d'un groupe à l'autre ou se situer entre plusieurs pôles. Une compréhension dynamique de ces pôles permet de saisir les enjeux de lutte de définition de la cause (il y a plusieurs manières de construire les violences des forces de l'ordre comme problème public) et les moments d'alliance ou de consensus pour une même perception de la cause ou un même mode d'action. Constitués de plusieurs collectifs, on pourrait exposer ces pôles ainsi : «autonomie » des familles ; organisation communautaire ; organisation des « premi.ère.ers concerné.e.s »; extrême-gauche anti-sécuritaire ; défense des droits de l'Homme.

\section{Pôle « autonomie » des familles de victimes}

Suite à la mort d'un habitant des quartiers populaires lors d'une intervention de police, un schéma tend à se reproduire : des habitant-e-s du même quartier, souvent ami-e-s de la famille, et des militant-e-s des quartiers populaires, se constituent en comité de soutien. Un même mot d'ordre revient, constituant souvent le nom du comité : Vérité et Justice pour la victime. Ces comités accompagnent les familles des victimes dans une mobilisation juridique ou politique lorsque cellesci ont lieu. Cela prend la forme de l'aide pour trouver un-e avocat-e, constituer et mener la plainte contre les agents de police ou de gendarmerie, lancer un appel à témoins et souvent mener une contre-enquête. Ces comités se mobilisent au plus près des familles des victimes (dont des membres font parfois partie), demandant une confiance réciproque, et revendiquent une mobilisation locale provenant des habitant-e-s du quartier de la victime. Pour ces militant-e-s, la volonté de la famille dans la manière de se mobiliser et son « autonomie » vis-à-vis de toute organisation politique sont au premier rang. Des militant-e-s des luttes de l'immigration post-coloniales et des quartiers populaires apportent ainsi leurs ressources militantes à ces familles de victimes et ces comités de

3 Bereni, L., « Quand la mise à l’agenda ravive les mobilisations féministes. L’espace de la cause des femmes et la parité politique (1997-2000) », Revue française de science politique 2009/2 (Vol. 59)

4 Mathieu, L., L'espace des mouvements sociaux, éditions du Croquant, coll « sociopo », 2012 
soutien. Les militant-e-s du Mouvement de l'Immigration et des Banlieues (MIB) contribuent fortement à développer ces modes d'action en se rendant auprès des familles de victimes, par la transmission de ressources militantes, et en développant un réseau entre familles de victimes et militant-e-s. Créé en 1995, le MIB s'inscrit dans l'héritage des luttes de l'immigration des années 1980, notamment la Marche pour l'égalité et contre le racisme de 1983, et du Comité contre la double peine dont il est issu. Ces militant-e-s habitant-e-s les quartiers populaires, présenté-e-s comme appartenant à la génération des « galériens » des cités ${ }^{5}$, acquièrent et développent un capital militant, revendiquant «l'auto-organisation » des habitant-e-s des quartiers populaires en tentant «d'articuler la dimension sociale et la dimension raciale de leur condition ${ }^{6}$ ». Les comités accompagnent souvent les recours juridiques d'actions collectives visant à faire connaître leur lutte et à tenter de s'imposer dans un rapport de force politique. Elles peuvent prendre la forme de marches, de réunions publiques, de sit-in, de rassemblements et d'événements de soutien (concerts, repas dans le quartier) pour alimenter la caisse de solidarité qui sert à payer les frais de justice. Ce militantisme cherche aussi à constituer un rapport de force devant ou au sein des tribunaux lors de procès, par exemple en occupant les bancs du public, liant ainsi dans un même lieu action en justice et combat politique. Le MIB a ainsi accompagné de nombreuses familles de victimes, et certains de ses anciens militants sont toujours actifs. Certaines mobilisations font date dans cette histoire militante : celle pour Youssef Khaïf, tué au Val-Fourré en 1991, durant laquelle le MIB et la famille dénoncent un « permis de tuer »; celles de d'Abdelkader Bouziane en 1997, puis de Xavier Dhem et Mohamed Berrichi en 2002 à Dammarie-les-Lys.

Vers la fin des années 2000, le MIB se dissout peu à peu, même si certain-e-s de ses militant-e-s restent actifs. Des familles de victimes créent à leur tour des collectifs pour à la fois sortir de l'isolement et proposer une mobilisation commune en rassemblant plusieurs familles ou comités de soutien : le collectif Vies Volées, créé en 2010 par Ramata Dieng, sœur de Lamine Dieng tué en 2007 suite à une clé d'étranglement lors d'une intervention de police ; le collectif Urgence notre police assassine (UNPA), créé en 2013 par Amal Bentounsi, sœur d'Amine Bentounsi tué en 2012 d'une balle dans le dos lors d'une intervention de police. Si ces comités entendent s'organiser au plus près des familles de victimes et prennent appui sur une mobilisation locale au sein du quartier, à partir du milieu des années 2000 une nouvelle génération de militant-es émerge, proposant davantage un mode d'organisation inspiré du community organizing provenant des États-Unis.

\section{Pôle organisation communautaire}

5 Hajjat, A., « Révolte des quartiers populaires, crise du militantisme et postcolonialisme », in Boubeker, A. et Hajjat, A. dir., Histoire politique des immigrations (post)coloniales... op.cit.

6 op.cit. p.249 
Dans les années 2000, des nouvelles politiques de la jeunesse se développent dans certaines municipalités, et cherchent à promouvoir un « pouvoir d'agir », c'est-à-dire la capacité des habitante-s des quartiers populaires à participer à la vie politique locale ayant comme enjeu principal l'apprentissage de la citoyenneté7. Des militant-e-s des quartiers populaires émergent, davantage intégré-e-s dans les structures politiques locales que la génération précédente, principalement en tant qu'éducateur-rice-s ou animateur-rice-s. Certains de ces collectifs naissent suite à des déviances des forces de l'ordre dans les quartiers populaires. Ces dernières sont perçues comme l'un des problèmes touchant les habitant-e-s des quartiers populaires. Ainsi, selon les méthodes de l'organisation communautaire, ce pôle se caractérise par l'action auprès ou au sein des institutions politiques, par la négociation avec les pouvoirs et partis politiques, afin de transformer les pratiques policières, ou du moins mettre à l'agenda politique la question des violences des forces de l'ordre à travers des propositions politiques : par exemple la revendication d'une meilleure formation des agents de police, la mise en place d'une police de proximité, ou différentes mesures administratives visant à un contrôle hiérarchique des pratiques des agents de terrain (caméras piétons, récépissé de contrôle d'identité, etc.). Contrairement au pôle précédent, la conception de l' «autonomie » politique inclut ici une coopération, même conflictuelle, avec les institutions et les partis parlementaires. Enfin, ce pôle se caractérise aussi par le fait que c'est la mobilisation politique auprès des institutions qui apparaît au premier plan et non pas l'action et le soutien auprès des familles ; ce qui n'exclut pas que ces organisations ou leurs membres participent par moments à des actions de soutien aux familles et que certain-e-s militant-e-s soient aussi investi-e-s dans des comités de soutien.

On peut prendre pour exemple la formation d'AClefeu, créé en 2005 à Clichy-sous-Bois après la mort de Zyed Benna et Bouna Traoré, et les brûlures de Muhitin Altun, et les rébellions qui ont suivi. La formation s'inscrit à la fois dans la dénonciation des déviances policières et dans la proposition d'un mode d'organisation des habitant-e-s des quartiers populaires, dont plusieurs de ses fondateur-rice-s ont été socialisé-e-s à la politique au sein du service jeunesse de Clichy-sous-Bois. AClefeu entend permettre aux habitant-e-s des quartiers populaires d'agir politiquement à travers l'écriture d'un cahier de doléances, l'apprentissage de la citoyenneté et la formation de «leaders des quartiers populaires », des actions en direction des institutions politiques, et le soutien de certain-e-s de leurs membres à se présenter sur les listes « autonomes » aux élections locales.

La référence au community organizing prend de plus en plus d'ampleur en France $^{8}$, et en

7 Hadj Belgacem, S., « Reconquérir la jeunesse des cités ? Enjeux et contradictions d'un Conseil Local des Jeunes dans une municipalité communiste », Mouvements, vol. 85, n¹, 2016

8 Talpin, J. et Balazard, H., « Community organizing: généalogie, modèles et circulation d'une pratique émancipatrice », Mouvements, vol.85, n²1, 2016 
2010 la campagne Stop le contrôle au faciès est lancée par des militant-e-s revenant d'une formation au community organizing aux États-Unis ${ }^{9}$. Cette campagne, qui rassemble plusieurs associations et collectifs, revendique l'instauration d'un récépissé lors des contrôles d'identités. Ces militant-e-s provenant des quartiers populaires sont en phase d'ascension sociale, bénéficiaient d'un plus fort capital scolaire que les générations précédentes, de ressources médiatiques, et de liens privilégiés avec des avocat-e-s, des Organisations Non Gouvernementales (ONG), et la Fondation Open Society. Le collectif s'est fortement investi dans un recours collectif déposé contre l’État en 2012 pour contrôles discriminatoires, qui aboutit le 24 juin 2015 à une condamnation de l'État français par la cours d'appel de Paris pour contrôles d'identité discriminatoires. Si certain-e-s de ces militante-s ont des liens privilégiés avec certaines familles de victimes, leur mobilisation se focalise sur des actions à visée nationale pour les habitant-e-s des quartiers populaires. Dans les mêmes années, des militant-e-s antiracistes apparaissent dans les mobilisations contre les violences des forces de l'ordre.

\section{Pôle organisation des « premier-ère-s concerné-e-s »}

Ces collectifs antiracistes se distinguent au sein de l'espace militant antiraciste par une primauté apportée aux victimes du racisme, ou les « premie-ère-s concerné-e-s ${ }^{10}$ ", et par un travail discursif dénonçant un « continuum colonial » amenant à une « surdétermination coloniale ${ }^{11}$ » dans l'explication des rapports de domination. Si ces collectifs ne prennent pas comme point de départ la dénonciation des violences des forces de l'ordre, on les retrouve dans cet espace militant à travers la dénonciation d'une critique post-coloniale des violences des forces de l'ordre, présentées comme provenant d'un « État raciste », colonial ou néo-colonial. Si des militant-e-s des autres pôles peuvent inclure une perspective post-coloniale, ce pôle se distingue par cette « surdétermination coloniale » de l'explication des violences des forces de l'ordre au détriment d'une dimension sociale comme dans d'autres pôles ; cette distinction étant à la source de nombreux conflits militants avec d'autres pôles. Ces militant-e-s proviennent principalement des quartiers populaires et sont héritier-ère-s de l'immigration post-coloniale, et tirent une partie de leurs ressources du fort capital scolaire, d'une proximité avec l'espace universitaire et l'espace médiatique de nombre d'entre eux-elles. Malgré une critique des médias dominants, ces militant-e-s mènent principalement des actions visant à interpeller le champ médiatique et le champ politique. On retrouve ainsi des militant-e-s du Parti des Indigènes de la République (PIR) ou encore de la Brigade Anti-Négrophobie (BAN). Si les premie-ère-s sont principalement visibles lors de grandes manifestations (comme les Marches de la

9 Carrel, M. et de Lépinay, A., « Expériences de community organizing en France », Mouvements, vol.85, n¹, 2016

10 Laplanche-Servigne, S., « Quand les victimes de racisme se mobilisent. Usage d'identifications ethnoraciales dans l'espace de la cause antiraciste en France et en Allemagne », Politix 2014/4, (n¹08)

11 Hajjat, A., « Révolte des quartiers populaires, crise du militantisme et postcolonialisme »... op.cit. 
Dignité du 31 octobre 2015 et du 19 mars 2017), les second-e-s sont davantage présent-e-s dans les mobilisations régulières contre les violences des forces de l'ordre et certain-e-s apportent aussi leurs ressources militantes pour l'organisation de manifestations de familles de victimes.

\section{Pôle extrême-gauche anti-sécuritaire}

Ce pôle militant comprend des collectifs portant une critique plus ou moins radicale des forces de l'ordre en tant qu'instrument d'oppression d’État au service du capitalisme. Cette critique accompagnant les aspirations de transformation radicale de la société, les militant-e-s de ce pôle proviennent de divers mouvements d'extrême-gauche (maoïstes, marxistes-révolutionnaires, anarchistes, ou «autonomes»), et se regroupent en collectif sur l'accord de positions antisécuritaires, ou anti-autoritaires. On retrouve ici davantage une dénonciation de l'institution policière en soi, perçue comme une institution de répression des classes populaires, mise en lien pour certains collectifs avec une répression des immigré-e-s et des héritier-ère-s de l'immigration post-coloniale. Ces collectifs agissent à travers l'organisation de débats publics, d'événements de soutien et caisses de solidarité aux victimes et familles de victimes, des publications de tracts, brochures et livres, l'organisation de manifestations. Mais on retrouve aussi plusieurs de ces militant-e-s auprès de familles de victimes ou dans des comités de soutien, apportant parfois des ressources militantes (publicisation des «affaires», contacts d'avocat-e-s, organisation de manifestation).

À titre d'exemple, le Collectif Angles Morts s'est créé à l'initiative de certain-e-s de ces militant-e-s habitant les quartiers populaires dans le cadre d'une mobilisation de soutien aux « cinq tireurs présumés » inculpés dans la répression des rébellions de Villiers-le-Bel en 2007 qui font suite à la mort de Lakhamy et Moushsin au cours d'une collision entre leur moto et une voiture de police. Ce collectif, liant lutte contre les violences des forces de l'ordre et lutte contre la répression carcérale, se mobilise dans des comité de soutien, organise des concerts de soutien, des réunions publiques, mais publie aussi des compte-rendus de procès ${ }^{12}$ ou des témoignages de militant-e-s et de familles de victimes ${ }^{13}$.

Aussi, dans les années 2000, avec l'utilisation de plus en plus régulière des Lanceurs de balles de défense (LBD - les plus connus sont les Flash-Ball) dans les quartiers populaires puis progressivement dans la répression de mouvements sociaux, de nouveaux collectifs de militant-e-s et victimes de ces armes émergent. On peut citer comme exemple le Collectif huit juillet et l'Assemblée des blessé-e-s. Le 8 juillet 2009, une manifestation contre la fermeture d'un squat à Montreuil est réprimée et cinq manifestant-e-s sont blessé-e-s par des tires de Flash-Ball, dont une 
personne qui perd un œil. Ces blessé-e-s et certain-e-s manifestant-e-s présent-e-s créent le Collectif huit juillet «pour préparer le procès contre les policiers, s’organiser avec d'autres personnes blessées et enquêter sur les pratiques et les armes de la police ${ }^{14}$ ». Les membres du collectif ont peu à peu pris contact avec d'autres blessé-e-s par Flash-Ball et des familles de victimes. Puis, en novembre 2014, suite à la mort de Rémi Fraisse ${ }^{15}$, à l'initiative du Collectif huit juillet se crée « l'Assemblée des blessé-e-s, des familles, des collectifs contes les violences policières », un réseau visant à créer une solidarité entre les blessé-e-s et leurs familles et à informer et lutter contre les violences des forces de l'ordre. Provenant de mobilisations étudiantes, écologistes, de soutien aux migrant-e-s, de squats, plusieurs collectifs se créent pour dénoncer la répression policière. Ces militant-e-s organisent des concerts de soutien, des réunions publiques, des manifestations. S'ils ne proviennent principalement pas des quartiers populaires, ils et elles créent du lien avec les familles de victimes et militant-e-s des quartiers populaires, notamment à travers l'organisation d'actions communes.

Enfin des réseaux se créent également pour entretenir une veille militante des cas de violences des forces de l'ordre ou de répression judiciaire et carcérale et des mobilisations qui émergent pour les dénoncer. Par exemple, le réseau Résistons ensemble contre les violences policières et sécuritaires, créé en 2002, publie un bulletin mensuel, sur leur site internet et en version papier qu'ils distribuent dans les quartiers populaires et dans les rassemblements militants. Pour finir, outre le recours au droit dans la mobilisation politique, on retrouve dans un cinquième pôle un usage du droit sous formes d'expertises servant la légitimation de la cause

\section{Pôle défense des droits de l'Homme}

Si le droit est un des modes d'action principalement utilisés par les familles de victimes qui portent plainte pour des déviances violentes des forces de l'ordre, des associations, des ONG, des avocat-e-s et magistrat-e-s se mobilisent également pour dénoncer un manque de respect des droits des individu-e-s par les forces de l'ordre. On retrouve des organisations telles que La Ligue des droits de l'homme (LDH), Amnesty International (AI), l'Association des chrétiens pour l'abolition de la torture (ACAT), ou encore des organisations de professionnel-le-s du droit, tels que le syndicat de la magistrature (SM) et le syndicat des avocats de France (SAF). Ces organisations de défense des droits s'attachent davantage à réaliser des enquêtes, qui donnent lieu à des publications de rapports, proposant ainsi un regard d'expertise sur les faits de déviances policières. Bénéficiant

14 Présentation du collectif huit juillet sur le site internet : https:/collectif8juillet.wordpress.com/

15 Dans la nuit du 25 au 26 octobre 2014, une manifestation a lieu sur le site prévu pour la construction du barrage de Sivens (dans le Tarn). Durant la manifestation, Rémi Fraisse, un militant écologiste, meurt de l'explosion d'une grenade offensive, coincée entre son sac à dos et son dos, tirée par une gendarme. 
d'une certaine légitimité auprès des médias, ces rapports et communiqués qui interpellent les autorités politiques contribuent à la reconnaissance politique et médiatique des déviances des forces de l'ordre. À titre individuel ou collectif, les membres de ces organisations apportent parfois leurs ressources juridiques et leur soutien à des familles de victimes dans la manière de mener l'action en justice et dans la publicisation de l'affaire. Mais ce n'est qu'à titre individuel que des avocat-e-s s'engagent auprès des familles de victimes dans une action en justice. Les rapports publiés tentent de rendre compte des types de déviances violentes des forces de l'ordre à travers des témoignages de victimes, un traitement quantitatif et un profilage des victimes à partir des plaintes recueillies par ces organisations. La commission Citoyens-Justice-Police, créée en 2002 par la LDH, le MRAP (Mouvement contre le racisme et pour l'amitié entre les peuples) et le SM, suite à une commission d'enquête spécifique sur «le comportement de policiers à Châtenay-Malabry, Poissy et Paris 20 ème $^{16}$ », est un des exemples de modes d'actions collectives ad-hoc de ces organisations. Ces enquêtes et rapports sont par moment repris par les familles de victimes et les autres groupes militants, servant d'outil de légitimation de leurs dénonciations des déviances des forces de l'ordre et d'une impunité policière.

\section{Conclusion}

D'autres initiatives, trop isolées ou spécifiques pour être présentes dans ces pôles ou pour constituer un pôle en soi, contribuent également directement ou indirectement à la construction d'une cause contre les violences des forces de l'ordre. On ne pourrait conclure cet article sans évoquer Maurice Rajsfus (Journaliste, historien, et militant contre les violences des forces de l'ordre) qui contribue à créer l'Observatoire des libertés publiques et le bulletin d'informations Que fait la police? en 1994 qui recense chaque mois, jusqu'en 2014, «toutes les informations concernant les petites et les grandes exactions des policiers ${ }^{17} »$. Ce bulletin d'information constitue une source précieuse, tant pour le militant que pour l'historien ou le sociologue, pour un recensement de cas de violences des forces de l'ordre en France. Aussi, des travaux de sociologues et politistes sur les pratiques policières contribuent également à créer une expertise sur les violences des forces de l'ordre, et leurs travaux constituent des sources « légitimes » reprises par les militante-s, telle que l'enquête Police et minorités visibles : les contrôles d'identité à Paris, publiée en $2009^{18}$.

Si ces modes de dénonciation des violences de forces de l'ordre présentés dans les cinq pôles

16 LDH, Commission d'enquête spécifique sur le comportement de policiers à Châtenay-Malabry, Poissy et Paris 20ème, 2002

17 Rajsfus, M., et Violet, A., Observatoire des libertés publiques. Que fait la police ?, éditions Dagorno, Paris, 1999

18 Jobard, F. et Lévy, R., Police et minorités visibles : les contrôles d'identité à Paris, Open Society Institut, 2009 
structurent cet espace militant, bien entendu, d'autres conceptions le traversent aussi telles que les modes de représentation et de dénonciation du racisme, la question du « racisme policier » étant aussi très présente et faisant l'objet de luttes. De même, il arrive que des individus et des collectifs soient positionnés dans différents pôles ici décrits, ou se déplacent d'un pôle à l'autre au fil de leurs trajectoires ou des phases de (re)configuration de l'espace de la contestation. L'étude de la construction d'une cause contre les violences des forces de l'ordre amène à considérer une diversité des trajectoires, des modes d'action et des ressources militantes, pouvant expliquer une diversité des causes qui traversent cet espace militant et qui dépassent la dénonciation des violences des forces de l'ordre. En effet, porter son attention sur les logiques sociales menant à la formation de ces mobilisations et sur les interactions entre les militant-e-s - comprenant que toute mobilisation ne va pas de soi - permet de saisir, à travers l'étude des modes d'action, les différentes perceptions de la question des violences des forces de l'ordre et les enjeux de lutte dans la définition d'une cause, ou d'un problème et des moyens de lutter contre.

Si certaines de ces mobilisations ont pu émerger dans le champ médiatique et politique, elles restent tout de même marginales pendant de longues années. Malgré la détention par des militant-es de ressources politiques et juridiques, cette marginalité s'explique notamment en raison d'une inégalité des ressources et d'accès aux espaces légitimes des médias et du monde politique. Elle s'explique aussi par la prégnance de représentations dominantes de ces champs. En raison d'une criminalisation des habitant-e-s des quartiers populaires et d'une tendance à l'intériorisation par les médias dominants des représentations policières, les victimes et leurs familles tendent à être criminalisées et leurs mobilisations délégitimées. Ainsi, l'un des principaux enjeux pour ces familles de victimes et de militant-e-s de différents pôles, en plus de la reconnaissance juridique des déviances des forces de l'ordre, est la reconnaissance médiatique et politique des victimes et des pratiques violentes des force de l'ordre. L'étude des conditions de possibilité de la reconnaissance de ces mobilisations amène à s'interroger sur le rôle joué par le recours à l'expertise et l'intervention publique d'acteurs ou d'actrices « légitimes » (professionnel-le-s de la politique, artistes, membres d'ONG, avocat-e et magistrat-e-s, sociologues et politistes), et sur la mise en avant dans le champ médiatique et le champ politique de nouvelles figures de victimes (Rémi Fraisse) et de fortes répressions de mouvements sociaux dans un contexte d'état d'urgence (COP21, Loi travail et Nuit Debout). Pour poursuivre la réflexion, on peut se demander si l'émergence dans les espaces légitimes de profils de victimes qui ne sont pas présentés comme appartenant aux franges les plus marginales et criminalisées de la population française (ce qui n'exclut pas des entreprises de criminalisation à travers la figure médiatique du « casseur ») ne contribue pas à une transformation des cadres de représentation médiatiques qui en retour aurait rendu possible un relatif accès au champ médiatique à des individus qui en étaient plus ou moins exclus auparavant (par exemple le 
comité Vérité pour Adama et le comité Justice pour Théo), indépendamment de leurs ressources militantes respectives. 